\title{
Breast Cancer Tumor Biology Characteristics and Its Correlation in a Tertiary Care Center
}

\author{
Tapan Kapoor ${ }^{1}$, Guman Singh ${ }^{2}$, Anushree Loyal ${ }^{3}$, Jyoti Sharma ${ }^{4}$, Dharam Pal Singh ${ }^{5}$
}

\begin{abstract}
Aims/Objectives: The study aims to evaluate and correlate the relationship between hormone receptor status-estrogen, progesterone receptor (ER and PR), and Herceptin receptor (Her-2/neu) with tumor characteristics in breast cancer patients.

Materials and methods: The current study included 101 breast cancer patients of various age-groups from the period 2016-2020. The tumors were evaluated for hormone expression, size, histologic grade, lymphovascular invasion, and axillary node status. The excised breast tissue specimen was evaluated for hormone status by immunohistochemistry (IHC) testing on paraffin blocks and was further analyzed using the fluorescence in situ hybridization (FISH) technique as per requirement. Stratification based on hormone receptor status was done in four categories-ER+PR+Her-2neu-, ER-PR-Her-2neu+, ER+PR+Her-2neu+, and ER-PR-Her-2neu-.

Results: In this study, 37.6\% of tumors were ER+PR+Her-2neu-, 13.8\% ER-PR-Her-2neu+, 13.8\% ER+PR+Her-2neu+, and 34.6\% ER-PR-Her2 neu-. Among high-grade tumors, more than half (52\%) patients were hormone receptor-negative, whereas among low-grade tumors $55 \%$ of patients were hormone receptor-positive. Sixteen percent of patients presented with a younger age-group ( $<35$ years) and in these patients, nearly $38 \%$ were Her- 2 /neu positive and most of the Her-2/neu receptor-positive patients ( $80 \%$ ) had lymphovascular space invasion. Primary tumor size at time of presentation was $<2 \mathrm{~cm}$ (T1) in only $11 \%$ of cases and $2-5 \mathrm{~cm}$ (T2) in $72 \%$ of cases. Lymph node positivity did not correlate with hormone receptor status.

Conclusion: The incidence of Her2-neu positive breast cancer patients was higher in the Indian population as compared to the Western world. The current study depicts the distinct shift in terms of tumor stage and biology, higher rate of Her-2/neu-positivity, younger age at diagnosis, larger tumor size, advanced tumor grade, and more positive lymph node involvement in contrast to the Western population.

Keywords: Breast cancer, Cancer, Histopathology.

Journal of Mahatma Gandhi University of Medical Sciences \& Technology (2021): 10.5005/jp-journals-10057-0143
\end{abstract}

\section{INTRODUCTION}

Worldwide, breast cancer is the most common malignancy affecting women and the leading cause of cancer death in most countries, with around 2.1 million newly diagnosed cases. Its incidence in Asia, Africa, South America, and other developing countries has experienced a steady rise in recent years, nearly contributing to almost one-third of the global disease burden and around 626,679 million deaths per year. ${ }^{1}$

In the Indian population, breast cancer is the most common cancer in women surpassing carcinoma cervix and affecting 178,361 new cases and 90,408 deaths reported annually. ${ }^{2}$ The encountered Indian population is usually younger ( $<40$ years), has a larger tumor size, and a higher grade at presentation as compared to the Western world. ${ }^{3}$

Recent reports suggest that there is an increase in the incidence of ER, PR, Her-2/neu negative (triple-negative), or Her-2/neu positive breast cancers which are biologically aggressive in nature, impervious to conventional cytotoxic chemotherapy treatment, and is associated with decreased overall survival (OS) compared to other subtypes of breast cancer. ${ }^{4-6}$

Her-2/neu (human epidermal growth factor receptor) is a tyrosine kinase receptor that is responsible for cell division, motility, growth, and repair. ${ }^{7,8}$ Its positivity denotes an aggressive disease phenotype, reduced disease-free survival (DFS), and OS. Therefore, its overexpression indicates a poor prognosis in early breast cancer patients. Trastuzumab (humanized monoclonal antibody) and other targeted anti-Her2 therapies have improved the outcome albeit it increases the financial burden as it is an expensive treatment. ${ }^{7,9,10}$ $\overline{{ }^{1-5} \text { Department of Radiation Oncology, Mahatma Gandhi Institute of }}$ Medical Sciences and Technology, Jaipur, Rajasthan, India

Corresponding Author: Tapan Kapoor, Department of Radiation Oncology, Mahatma Gandhi Institute of Medical Sciences and Technology, Jaipur, Rajasthan, India, Phone: +91 7597783390, e-mail: tk2909@gmail.com

How to cite this article: Kapoor T, Singh G, Loyal A, et al. Breast Cancer Tumor Biology Characteristics and Its Correlation in a Tertiary Care Center. J Mahatma Gandhi Univ Med Sci Tech 2021;6(2):53-55.

Source of support: Nil

Conflict of interest: None

This retrospective institutional study aimed to analyze hormone and Her-2/neu receptor status along with other tumor characteristics and to ascertain the correlation between these tumor characteristics in Indian breast cancer patients.

\section{Materials and Methods}

This retrospective study included 101 histopathologically proven invasive breast cancer patients who presented or were referred to Oncology OPD at our tertiary care center from the year 2016 to 2020. Estrogen receptor, PR, and Her-2/neu receptor status done via immunohistochemistry (IHC) and fluorescence in situ hybridization (FISH), demographic as well as other histopathological tumor characteristics including age, tumor size, Bloom-Richardson grade, axillary lymph node status, lymphovascular space invasion were 
retrieved from Hospital Electronic Medical Records System as well as offline patient record. Estrogen receptor, $\mathrm{PR}$, and Her-2/neu receptor $\mathrm{IHC}$ were done using the immunoperoxidase method on paraffin blocks of the tumor. ${ }^{11}$ For ER and PR, a scale of $1-3$ was used and the scoring was done using the Shousha method, which depended on the percentage of stained cells, where score 1 indicated $<33 \%$, score 2 depicted $33-66 \%$, and score 3 showed $>66 \%$ of positive nuclei. ${ }^{11-13}$ Similarly, staining of the nucleus was graded as mild (score 1), moderate (score 2), and intense (score 3). Estrogen receptor and PR score are given by multiplication of these two scores (area $x$ intensity). A minimum score of 1 was categorized as positive and the maximum possible score was 9. HER2 scoring was done on IHC slides as per the American Society of Clinical Oncology (ASCO) guidelines 2007. Score 0 and score 1 were considered as negative, whereas score 2 was equivocal (further assessed by FISH), and score 3 was regarded as positive. ${ }^{11,12}$

\section{Results (Tables 1 to 8 )}

Table 1: Receptor status and the percentage of positivity

\begin{tabular}{lll}
\hline Group & Hormone status & Percentage \\
\hline I $(n=38)$ & ER/PR+HER-2- & 37.6 \\
II $(n=14)$ & ER/PR-HER-2+ & 13.8 \\
III $(n=14)$ & ER/PR+HER-2+ (TPBC) & 13.8 \\
IV $(n=35)$ & ER/PR-HER-2- (TNBC) & 34.6 \\
\hline
\end{tabular}

Overall, there was a marginal difference between the incidence of ER, $P R$ positive and ER, PR negative receptor status with a slight inclination toward positivity

Table 2: Correlation of receptor status with age

\begin{tabular}{lllll}
\hline & & $E R / P R-$ & $E R / P R+$ & \\
& ER/PR+ & HER-2- & HER-2+ & ER/PR- \\
Age & HER-2-(\%) & $($ TNBC) (\%) & $($ TPBC) $(\%)$ & HER-2+ (\%) \\
\hline$<35(n=16)$ & 36 & 4 & 44 & 16 \\
$36-50(n=45)$ & 32.5 & 39.5 & 16.2 & 11.6 \\
$>50(n=40)$ & 33.3 & 38.4 & 15.3 & 12.8 \\
Total & & & & \\
\hline
\end{tabular}

Most patients (60.3\%) were $<50$ years and were ER, PR, and HER-2 positive (TPBC-44\%)

Table 3: Relationship of grade with the receptor status

\begin{tabular}{|c|c|c|c|c|}
\hline Grade & $\begin{array}{l}E R / P R+ \\
H E R-2-(\%)\end{array}$ & $\begin{array}{l}E R / P R-H E R- \\
2-(T N B C) \\
(\%)\end{array}$ & $\begin{array}{l}E R / P R+ \\
H E R-2+ \\
(T P B C)(\%)\end{array}$ & $\begin{array}{l}E R / P R-H E R- \\
2+(\%)\end{array}$ \\
\hline I $(n=6)$ & 50 & 16.67 & 33.3 & 0 \\
\hline II $(n=46)$ & 54.3 & 17.3 & 8.6 & 19.5 \\
\hline III $(n=49)$ & 20.4 & 51.02 & 18.3 & 10.2 \\
\hline
\end{tabular}

Higher-grade tumor (grade III) was more associated with triple-negative breast cancer whereas low- and intermediate-grade tumors were mostly hormone receptor-positive

Table 4: Correlation of tumor size with hormone receptor status

\begin{tabular}{|c|c|c|c|c|}
\hline $\begin{array}{l}\text { Tumor size } \\
(\mathrm{cm})\end{array}$ & $\begin{array}{l}E R / P R+ \\
H E R-2-(\%)\end{array}$ & $\begin{array}{l}E R / P R-H E R- \\
2-(T N B C) \\
(\%)\end{array}$ & $\begin{array}{l}E R / P R+ \\
H E R-2+ \\
(T P B C)(\%)\end{array}$ & $\begin{array}{l}E R / P R-H E R- \\
2+(\%)\end{array}$ \\
\hline$<2(n=10)$ & 70 & 0 & 30 & 0 \\
\hline $2-5(n=62)$ & 35.4 & 38.7 & 12.9 & 12.9 \\
\hline$>5(n=29)$ & 27.5 & 34.4 & 17.2 & 20.6 \\
\hline
\end{tabular}

Approximately $60 \%$ of our patients had tumor size of the range of $2-5 \mathrm{~cm}$ and there was no correlation between hormone receptor status and tumor size
Table 5: Relationship of lymphovascular space invasion with the receptor reactivity

\begin{tabular}{lllll}
\hline & & $E R / P R-$ & $E R / P R+$ & \\
& $E R / P R+$ & $H E R-2-$ & $H E R-2+$ & $E R / P R-$ \\
LVSI & $H E R-2-(\%)$ & $(T N B C)(\%)$ & $(T P B C)(\%)$ & $H E R-2+(\%)$ \\
\hline Present $(n=58)$ & 34.4 & 36.2 & 20.6 & 8.6 \\
Absent $(n=43)$ & 51.1 & 39.5 & 4.6 & 4.6 \\
\hline
\end{tabular}

Lymphovascular space invasion was seen in $57 \%$ of our patients, and out of them, $44.8 \%$ of patients were hormone receptor-negative. Lymphovascular space invasion was absent in more than half (51.1\%) of ER, PR positive patients.

Table 6: Correlation of axillary lymph node with hormone receptor status

\begin{tabular}{lllll}
\hline & & $E R / P R-$ & $E R / P R+$ & \\
& $E R / P R+$ & $H E R-2-$ & $H E R-2+$ & $E R / P R-$ \\
& $H E R-2-(\%)$ & $(T N B C)(\%)$ & $(T P B C)(\%)$ & $H E R-2+(\%)$ \\
\hline $\begin{array}{l}\text { Positive }(n= \\
78)\end{array}$ & 35 & 32.5 & 16.25 & 13.7 \\
Absent $(n=23)$ & 33.3 & 38.09 & 14.2 & 14.2 \\
\hline
\end{tabular}

In this study, lymph node involvement was seen in $77 \%$ of patients, and out of them, $35 \%$ were ER/PR positive and $32.5 \%$ were ER/PR negative

Table 7: Association of HER-2 receptor status with age

\begin{tabular}{ll}
\hline Age & HER-2 positive cases (\%) \\
\hline$<35(n=6)$ & 20.6 \\
$36-50(n=12)$ & 41.3 \\
$>50(n=11)$ & 37.9 \\
\hline
\end{tabular}

Maximum patients $<50$ years of age were HER-2 positive (61.9\%)

Table 8: Association of HER-2 positivity with grade

\begin{tabular}{lc}
\hline Grade & HER-2 positive cases (\%) \\
\hline I $(n=2)$ & 6.8 \\
II $(n=13)$ & 44.8 \\
III $(n=14)$ & 48.2 \\
\hline
\end{tabular}

The intermediate- and high-grade tumors had an almost equal association with HER-2 positivity status

\section{Discussion}

The current retrospective study aimed to identify the relationship between hormone receptor status with other tumor characteristics in breast cancer patients. After considering many points of note, these data are different from the corresponding Western literature. Around $60 \%$ of our patients belong to $<50$ years and among those, $16 \%$ belong to $<35$ years of the age-group which is in contrast to the Western world where maximum cases belong to $>50$ years of age-group. ${ }^{3,4}$ Similar to other Indian population-based studies, this study also demonstrated the peak age of breast cancer presentation was between 35 and 50 years (44.5\%). ${ }^{3,4}$ The study showed an association of higher grade tumors with triple-negative breast cancer, whereas low- and intermediate-grade tumors were hormone receptor-positive. .,4 $^{3}$

In this study, ER/PR hormone expression was $51.4 \%$ which is significantly less than the western population having approximately $65 \%$ of hormone receptor positivity, whereas a total of $27.4 \%$ of cases were HER-2 positive which is slightly greater than HER-2 positive cases (around 18-20\%) in the Western literature. $3,14,15$ 
Triple-negative (TNBC) hormone receptor status in this study was also significantly higher (34.6\%) as compared to the western world (12-13\%). ${ }^{16}$

Our findings also suggest that HER2-positive breast cancer is more associated with higher grade (grade II/III) tumors. Overexpression of HER2 was almost equally associated with ER, PR positive, and negative receptor status. Estrogen receptor/PR-Her-2 neu positive tumor subtype is more common among young Indian females that have a poorer prognosis as compared to hormone receptor-positive tumors., ${ }^{7,15}$

Breast cancer screening programs enabling breast cancer detection at an early age have distinct tumor stages and biology, low tumor grade, smaller tumor size, less lymph node involvement, and a lower rate of HER-2 positivity. In developing countries, the majority of breast cancers are detected at a later stage, thereby having aggressive nature. ${ }^{7}$

Thus, in the current study (based on the North-West Indian population), HER2-positive breast cancer incidence was slightly higher, whereas ER/PR was significantly less as compared to the incidence in the Western population. Triple-negative hormone receptor status was also significantly higher (34.6\%) as compared to the western world (12-13\%). Other key observations of this study suggested that breast cancer in Indian women presents at an earlier age, with a higher histological grade and unfavorable hormone receptor status thus having a poorer prognosis.

\section{References}

1. Bray F, Ferlay J, Soerjomataram I, et al. Global cancer statistics 2018: GLOBOCAN estimates of incidence and mortality worldwide for 36 cancers in 185 countries. CA Cancer J Clin 2018;68(6):394-424. DOI: 10.3322/caac.21492.

2. Globocan 2018: India Factsheet. http://cancerindia.org.in/globocan2018-india-factsheet.

3. Desai SB, Moonim MT, Gill AK, et al. Hormone receptor status of breast cancer in India: a study of 798 tumours. The Breast 2000;9(5):267-270. DOI: 10.1054/brst.2000.0134.

4. Kakarala M, Rozek L, Cote M, Liyanage S, et al. Research article breast cancer histology and receptor status characterization in Asian Indian and Pakistani women in the U.S. - a SEER analysis. BMC Cancer 2010;10(1):191. DOI: 10.1186/1471-2407-10-191.
5. Deapen D, Liu L, Perkins C, et al. Rapidly rising breast cancer incidence rates among Asian-American women. Int J Cancer 2002;99(5):747-750. DOI: 10.1002/ijc.10415.

6. Lin CH, Liau JY, Lu YS, et al. Molecular subtypes of breast cancer emerging in young women in Taiwan: evidence for more than just westernization as a reason for the disease in Asia. 2009;18(6):18071814. DOI: 10.1158/1055-9965.EPI-09-0096.

7. Pathmanathan N, Geng J, Li W, et. al. Human epidermal growth factor receptor 2 status of breast cancer patients in Asia: results from a large, multicountry study. Asia-Pacific J Clin Oncol 2016;12(4):369-379. DOI: 10.1111/ajco.12514.

8. Yarden Y, Sliwkowski MX. Untangling the ErbB signalling network. Nat Rev Mol Cell Biol 2001;2(2):127-137. DOI: 10.1038/35052073.

9. Weigel MT, Dowsett M. Current and emerging biomarkers in breast cancer: prognosis and prediction. Endocr Relat Cancer 2010;17(4):R245-R262. DOI: 10.1677/ERC-10-0136.

10. Press MF, Pike MC, Chazin VR, et al. Her-2/neu expression in nodenegative breast cancer: direct tissue quantitation by computerized image analysis and association of overexpression with increased risk of recurrent disease. Cancer Res 1993;53(20):4960-4970.

11. Gupta $S$, Shet T, Suryavanshi P, et al. Estrogen, progesterone and HER2 receptor expression in breast tumors of patients, and their usage of HER2-targeted therapy, in a tertiary care centre in India. Indian J Cancer 2011;48(4):391-396. DOI: 10.4103/0019-509X.92245.

12. Shousha S, Coady AT, Stamp T, et al. Oestrogen receptors in mucinous carcinoma of the breast: an immunohistological study using paraffin wax sections. J Clin Patho 1989;42(9):902-905. DOI: 10.1136/ jcp.42.9.902.

13. Akiyama F, Iwase H. Triple negative breast cancer: clinicopathological characteristics and treatment strategies. Breast Cancer 2009;16(4):252253. DOI: $10.1007 / \mathrm{s} 12282-009-0140-x$.

14. Vedashree M, Rajalakshmi V. Clinico-pathological study of breast carcinoma with correlation to hormone receptor status and HER-2/ neu. Indian J Pathol Oncol 2016;3(4):690-695. DOI: 10.5958/23946792.2016.00128.9.

15. Lal P, Tan LK, Chen B. Correlation of HER-2 status with estrogen and progesterone receptors and histologic features in 3,655 Invasive breast carcinomas. Am J Clin Pathol 2005;123(4):541-546. DOI: 10.1309/YMJ3A83TB39MRUT9.

16. Kulkarni A, Kelkar DA, Parikh N, et al. Meta analysis of prevalence of triple-negative breast cancer and its clinical features at incidence in Indian patients with breast cancer. JCO Global Oncol 2020;6(6):10521062. DOI: $10.1200 / G O .20 .00054$. 